Vol.: 4 Issue: 2 Date: 31.12.2020 Received: 12.06.2020 Accepted: 10.09.2020 Final Version: 16.10.2020

ISVOS Journal, 2020, 4(2): 81-85 - https://doi.org/10.47897/bilmes.751995

\title{
IoT Based Indoor Disinfection Coordinating System Against the New Coronavirus
}

\author{
Firat Aydemir a,1 \\ ${ }^{a}$ Kütahya Dumlupınar University, Computer Engineering Department, Türkiye \\ ORCID ID: 0000-0002-8965-1429
}

\begin{abstract}
In this study, a system solution for monitoring and coordinating indoor disinfection processes based on the Internet of Things technology is presented. Studies about COVID-19 shows that novel coronavirus is spreading through the virus-containing droplets exhaled by infected people on the surfaces; moreover, it is shown that the virus can remain stable up to 72 hours depending on the type of surface. Therefore, proper sterilization and disinfection routines in public areas play a major role in reducing the spread of coronavirus. In the proposed conceptual system, IoT nodes, consisting of single-board computer and camera, separate the human density in certain regions into various levels through image processing algorithms and write these densities in a cloud database. An Android application reads data from the cloud database periodically and locates the risky areas on the map. When the sterilization staff disinfects the specified spots, his/her location is determined in the android application via Bluetooth beacons located in the area, and the database is updated to show that disinfection is complete in these areas.
\end{abstract}

Keywords: "Internet of things, COVID-19, disinfection, bluetooth beacon, cloud database"

\section{Introduction}

Since December 2019, the new coronavirus which is named as 2019-nCoV by the World Health Organization (WHO), has rapidly spread from Wuhan, China's Hubei province to other parts of China, and then worldwide [1]. The disease caused by the 2019-nCoV virus, COVID-19, was identified as a pandemic on March 11 due to the spread and severity of the virus in 113 countries, except in China [2]. The number of known coronavirus infections around the world has been reached 6.5 million and more than 350 thousand people have died from the disease [3].

Countries have taken a series of measures to prevent the spreading of the virus; initial measures such as shutting down borders, curfews, halting domestic and international flights were followed by the cancellation of many events, including sports tournaments, concerts, and festivals. Every interaction between people increases the risk of spread; therefore, in many cities, malls, restaurants, coffee shops, and public parks and gardens have also been closed to minimize the interaction. Because the person-to-person transmission is primarily occurred through direct contact or by droplets that spread through the infected person's sneeze or cough [4-6].

After four months of the declaration of the COVID-19 epidemic as a public health emergency of international concern by WHO [7]; despite the significant number of patients and casualties, in many countries, governments have started or are planning to gradually relax restrictions because of the economic impacts of coronavirus. In the first place, shopping centers, restaurants, bars, cafes, hairdressers have been allowed to restart business considering low capacity and social distance. However, there is always the risk that the second wave, as in the case of Singapore, Japan, and Iran, in which new cases increase, may emerge after restrictions were loosened.

At this point, we need to make more use of technology to prevent possible second and third waves of the COVID-19 infection. The Internet of Things (IoT), defined as interconnected smart devices that are changing data without any human interaction at any level through a defined network, is one of the key technologies that can be used during the COVID-19 pandemic [8]. IoT based systems can present effective solutions to prevent, control, and monitor infectious diseases [9].

\footnotetext{
${ }^{1}$ Corresponding author. 0 (274) 4434254 / 4269

E-mail address: firat.aydemir@dpu.edu.tr
} 
In this study, we present a monitoring and coordinating system solution for disinfection processes at public enclosed areas that is based on the IoT to prevent the spreading of the 2019-nCoV virus. (See Figure 1) Effective and proper disinfection will be helpful in the fight against the spread of the virus. The parts of the paper are arranged as follows; section II presents a detailed literature survey about the transmission path of the new virus, prevention and inactivation methods, and also IoT-based studies against the virus. The proposed methodology is explained in section III. The conclusions and further discussions on this study are given in section IV.

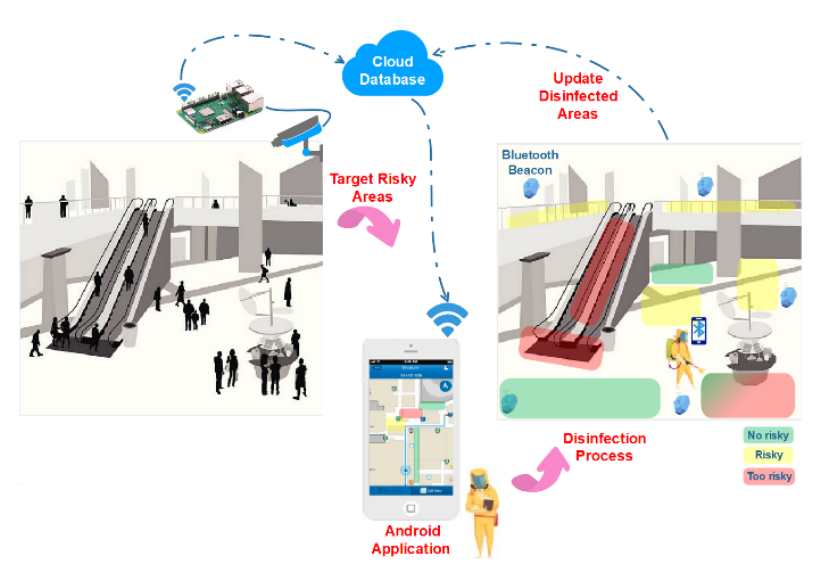

Figure 1.The proposed solution, an example application for shopping mall disinfection

\section{Related Work}

Preliminary information about the contamination of coronavirus is mentioned above. In this section, detailed information about the spreading of the virus, inactivation methods for the virus, and protective measures in the literature are mentioned; and then, studies based on the IoT technology are emphasized in the struggle with Covid-19.

\subsection{About 2019-nCoV virus: transmission, prevention, and inactivation}

The novel coronavirus which typically causes respiratory and gastrointestinal sickness has become a global health threat for humanity [10]. In order to stop this threat, it is necessary to understand clearly how the virus spreads. Unfortunately, the coronavirus is highly contagious, and there are still some uncertain spots about virus transmission.

The virus can spread via direct and indirect contact. As mentioned earlier, human-to-human transmission occurs mainly via droplets that spread through the patients' sneeze, cough, or even talk. These contagious droplets can reach up to $2 \mathrm{~m}$ distance and remain infectious for up to 9 days $[6,11]$. The droplets that contain the virus in most cases drop to the floor or a surface in a short time. However, some part of these droplets whose diameter are less than five microns can remain in the air for three hours; this finding indicates that being in the same room with an infected person increases the probability of aerosol transmission of the virus. The droplets containing viruses on different kinds of surfaces remain contagious for different durations; such as, on plastic surfaces for 72 hours, on stainless steel for 48 hours, on copper for 4 hours $[12,13]$. Nevertheless, the persistence of the 2019$\mathrm{nCoV}$ virus on inanimate surfaces reduces with the increasing temperature. For instance, at $4^{\circ} \mathrm{C}$ the virus is stable and remains viable up to 28 days, although, when the temperature rises to $70^{\circ} \mathrm{C}$, the virus becomes inactive in 5 min. [11, 14]. The transmission due to the droplets on inanimate surfaces may occur if a person touches these infected surfaces, and then making direct contact with his/her nose, mouth, or even eyes with hands [15, 16].

The only way to stop the spreading of infection is to prevent contact with infected people. However, this is not a realistic solution when it is considered that there are asymptotic carriers of the virus and infected individuals during the incubation period who do not show any symptoms. Therefore, personal protection should be taken by wearing a mask, observing social distancing rules, paying attention to self-hygiene, and so on.

Environmental disinfection plays an important role in killing infectious agents such as viruses and bacteria. The new coronavirus can be inactivated with surface disinfection procedure by using $0.1 \%$ sodium hypochlorite, $0.5 \%$ hydrogen peroxide, or $62-71 \%$ ethanol for 1 min duration [17]. 


\subsection{The Internet of Things: fight against pandemic}

Application areas such as wearable devices, smart cities, smart healthcare, smart agriculture, etc., and market share of the Internet of Things technology are increasing day by day. During this pandemic, IoT based health monitoring systems, and wearable devices can be used efficiently against the COVID-19 with minor revisions. Unlike the rest of the world, China used IoT based solutions very effectively to stop spreading the virus; for example, location-based services and big data analytics were used to identify high-risk cases.

Due to health issues and restrictions worldwide, studies in the literature specifically about new coronavirus based on IoT mostly consisted of reviews (by the time I searched the web in May 2020). However, there are still some new approaches that could be found in the literature.

A system that operates on an edge computation platform using a thermal camera and Raspberry Pi camera can measure vital parameters of patients such as heart rate, respiratory effort and detect fever in the emergency department to decrease the triage durations, although the system needs to be optimized for real-life usage [18].

An integrated software and hardware platform which uses IoT based sensors to monitor patient physiologic conditions and mechanical ventilator operations was proposed [19]. The developed prototype device consists of Raspberry Pi that can be attached to the mechanical ventilator to collect the patient's ventilation data and another IoT device that collects patient blood oxygenation data. After collecting and storing data in a database, analytic algorithms are applied for diagnosis purposes.

An Internet of Medical Things enabled wrist-worn device was proposed to sense and track the same devices within its range [20]. The main idea is to warn people about the mildly and highly suspicious individuals in a nearby area, and also via LED lighting indicate the current condition of the wearer. The Bluetooth connection and Wi-Fi connection were used for inter-device communication and communication with the health authorities respectively.

\section{Methodology and Design Example}

The block diagram of the proposed IoT based monitoring and coordinating system for indoor disinfection is presented in Figure 2. The system consists of four main structures; IoT nodes, an Android application, a cloud database, and Bluetooth beacons.

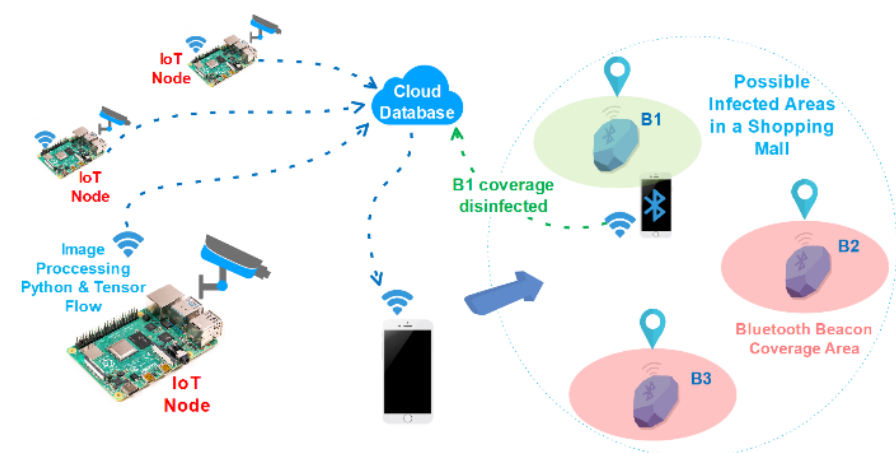

Figure 2. The block diagram of the proposed solution

A single IoT node that is consisting of a Raspberry Pi and a camera module detects and tracks people in the camera's field of view, and stores the data into the cloud database. Free open-source image processing library, OpenCV, is chosen with Python code. The frame in the camera's field of view is divided into sectors according to the Bluetooth beacons located inside of it. Raspberry Pi connects to the Internet over Wi-Fi. The screenshot of the human detection and tracking application performed on a sample video taken from a shopping center is shown in Figure 3. 


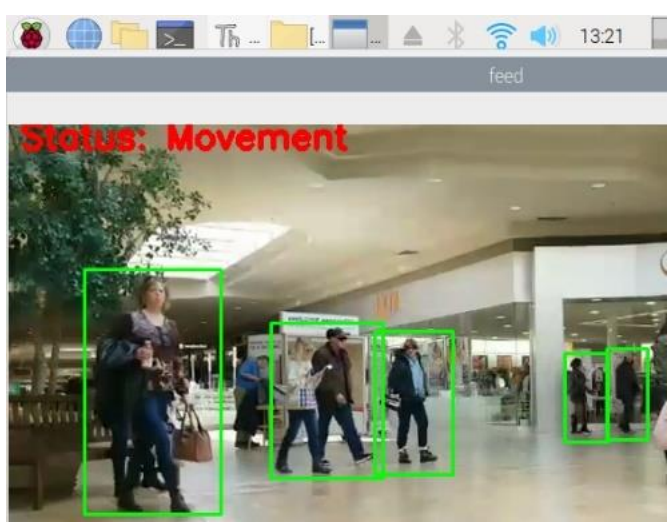

Figure 3. Sample application of human detection

The database can be updated by IoT nodes and Android application which runs on disinfection staffs' mobile phone. Each IoT node can increase the risk to higher levels for the sectors in the area according to crowd density; in contrast to the IoT nodes, the Android application works towards reducing risk to lower levels by updating the database whenever staff disinfects these sectors. The only components that are not mandatory to use in the proposed system are the IoT nodes. Because in many enclosed areas, images obtained from security cameras can be subjected to image processing on a PC at the server room or security room, and then the database can be updated; such a change also reduces the overall cost of the system.

The Android application periodically reads data from the database to show the risk levels on the map. When the sterilization staff goes to risky areas and spends enough time to perform disinfection processes, the application updates the database as reducing the risk level of that specific region. If the staff postpones the sterilization, he/she gets notifications via the application periodically.

The Bluetooth beacons use Bluetooth Low Energy (BLE) technology which is energy efficient and has lower costs than standard Bluetooth devices. The transmission ranges of BLE are very short typically several meters that make BLE a good choice for indoor localization applications [21-23]. By considering the shopping mall example; the IDs of the beacons already used by the stores in the shopping malls can be introduced to the system to determine indoor localization. If there are not enough beacons in the mall, the stores can be encouraged to buy these products, thus they can contribute to the determination of sterilization staff location as well as increase their sales by reaching their customers easily.

In addition to the components mentioned in the system, by using another Android application customers can scan a data matrix code at the entrance of a shopping mall and get information about risky places inside of the mall; thus they can avoid spending too much time at places where the disinfection process has not been completed.

There are also economic advantages of using that kind of IoT based disinfection monitoring system, such as; the municipalities that are responsible for carrying out disinfection of public areas allocate serious budgets; if they can achieve to use disinfectant products only in the required areas, they will contribute to the economy.

\section{Conclusion}

One of the best ways to stop the spread of the coronavirus is to isolate anyone who is infected by the virus. Unfortunately, this is not a practical solution since without testing it is not possible to know who is infected and who is not, especially considering that some of the infected people do not show symptoms. So, the best thing to do is people need to be more careful about cleaning than they always do. In addition to personal cleaning, the disinfection, and sterilization processes of public areas, particularly indoor locations should be done properly. The proposed IoT based monitoring and coordinating system solution for disinfection processes could provide better hygiene in enclosed areas. By using image processing the IoT nodes in the system categorize the enclosed areas according to the risk conditions and provide better hygiene by ensuring that the necessary locations are sterilized. The Bluetooth beacons placed in certain points are used to locate the disinfection staff position. Via the Android application, the risk situations of the disinfected areas are updated in the database. When the proposed system solution will be concluded, it will provide better cleaning as well as economical savings by ensuring the effective usage of disinfectant products.

\section{References}

[1] N. Zhu et al., “A novel coronavirus from patients with pneumonia in China, 2019,” N. Engl. J. Med., vol. 382, no. 8, pp. 727-733, 2020. 
[2] World Health Organization (2020) Novel Coronavirus (2019-nCoV). Situation Report-51, 11 March 2020.

[3] World Health Organization (2020) Novel Coronavirus (2019-nCoV). Situation Report-137, 5 June 2020.

[4] H. A. Rothan and S. N. Byrareddy, "The epidemiology and pathogenesis of coronavirus disease (COVID-19) outbreak," J. Autoimmun., vol. 109, no. February, pp. 18-21, 2020.

[5] Y. H. Jin et al., "A rapid advice guideline for the diagnosis and treatment of 2019 novel coronavirus (2019-nCoV) infected pneumonia (standard version),” Med. J. Chinese People’s Lib. Army, vol. 45, no. 1, pp. 1-20, 2020.

[6] T. Singhal, “A Review of Coronavirus Disease-2019 (COVID-19),” Indian J. Pediatr., vol. 87, no. 4, pp. 281-286, 2020.

[7] World Health Organization (2020) Novel Coronavirus (2019-nCoV). Situation Report-11, 31 January 2020.

[8] R. P. Singh, M. Javaid, A. Haleem, and R. Suman, "Internet of things (IoT) applications to fight against COVID-19 pandemic," Diabetes Metab. Syndr., vol. 14, no. 4, pp. 521-524, 2020.

[9] E. Christaki, "New technologies in predicting, preventing and controlling emerging infectious diseases," Virulence, vol. 6 , no. 6 , pp. 558-565, 2015.

[10] M. Pal, G. Berhanu, C. Desalegn, and V. Kandi, "Severe Acute Respiratory Syndrome Coronavirus-2 (SARS-CoV-2): An Update," Cureus, vol. 2, no. 3, 2020.

[11] G. Kampf, D. Todt, S. Pfaender, and E. Steinmann, "Persistence of coronaviruses on inanimate surfaces and their inactivation with biocidal agents,” J. Hosp. Infect., vol. 104, no. 3, pp. 246-251, 2020.

[12] L. Patients, D. Taylor, A. C. Lindsay, and J. P. Halcox, "Aerosol and Surface Stability of SARS-CoV-2 as Compared with SARS-CoV-1,” N. Engl. J. Med., pp. 0-3,2020.

[13] Y. Y. Liu et al., "Aerodynamic Characteristics and RNA Concentration of SARS-CoV-2 Aerosol in Wuhan Hospitals during COVID-19 Outbreak," bioRxiv, vol. 86, no. 21, p. 2020.03.08.982637, 2020.

[14] A. W. H. Chin et al., "Stability of SARS-CoV-2 in different environmental conditions," The Lancet Microbe, vol. 1, no. 1, p. e10, 2020.

[15] S. P. Adhikari et al., "Epidemiology, causes, clinical manifestation and diagnosis, prevention and control of coronavirus disease (COVID-19) during the early outbreak period: A scoping review," Infect. Dis. Poverty, vol. 9, no. 1, pp. 1-12, 2020.

[16] C. wei Lu, X. fen Liu, and Z. fang Jia, "2019-nCoV transmission through the ocular surface must not be ignored," Lancet, vol. 395, no. 10224, p. e39, 2020.

[17] G. Pascarella et al., "COVID-19 diagnosis and management: a comprehensive review”, vol. 2019. 2020.

[18] C. Hegde et al., "AutoTriage - An Open Source Edge Computing Raspberry Pi-based Clinical Screening System," medRxiv, p. 2020.04.09.20059840, 2020.

[19] G. B. Rehm, X. L. Chen, B. T. Kuhn, I. Cortes-puch, N. R. Anderson, and J. Y. Adams, "Leveraging IoTs and Machine Learning for Patient Diagnosis and Ventilation Management in the Intensive Care Unit," pp. 1-11, 2020.

[20] A. K. Tripathy, A. G. Mohapatra, S. P. Mohanty, E. Kougianos, A. M. Joshi, and G. Das, "EasyBand: A Wearable for Safety-Aware Mobility during Pandemic Outbreak,” IEEE Consum. Electron. Mag., pp. 1-5, 2020.

[21] S. I. Sou, W. H. Lin, K. C. Lan, and C. S. Lin, "Indoor location learning over wireless fingerprinting system with particle markov chain model,” IEEE Access, vol. 7, pp. 8713-8725, 2019.

[22] Y. Zhuang, J. Yang, Y. Li, L. Qi, and N. El-Sheimy, "Smartphone-based indoor localization with bluetooth low energy beacons," Sensors (Switzerland), vol. 16, no. 5, pp. 1-20, 2016.

[23] P. Kriz, F. Maly, and T. Kozel, ““'Improving Indoor Localization Using Bluetooth Low Energy Beacons”, Mob. Inf. Syst., vol. 2016, 2016. 